\title{
New Optimality Conditions for a Nondifferentiable Fractional Semipreinvex Programming Problem
}

\author{
Yi-Chou Chen ${ }^{1}$ and Wei-Shih Du ${ }^{2}$ \\ ${ }^{1}$ Department of General Education, National Army Academy, Taoyuan 320, Taiwan \\ ${ }^{2}$ Department of Mathematics, National Kaohsiung Normal University, Kaohsiung 824, Taiwan
}

Correspondence should be addressed to Wei-Shih Du; wsdu@nknucc.nknu.edu.tw

Received 29 July 2012; Accepted 1 January 2013

Academic Editor: Jen Chih Yao

Copyright (C) 2013 Y.-C. Chen and W.-S. Du. This is an open access article distributed under the Creative Commons Attribution License, which permits unrestricted use, distribution, and reproduction in any medium, provided the original work is properly cited.

We study a nondifferentiable fractional programming problem as follows: $(P) \min _{x \in K} f(x) / g(x)$ subject to $x \in K \subseteq X$, $h_{i}(x) \leq$ $0, i=1,2, \ldots, m$, where $K$ is a semiconnected subset in a locally convex topological vector space $X, f: K \rightarrow \mathbb{R}, g: K \rightarrow \mathbb{R}$ and $h_{i}: K \rightarrow \mathbb{R}, i=1,2, \ldots, m$. If $f,-g$, and $h_{i}, i=1,2, \ldots, m$, are arc-directionally differentiable, semipreinvex maps with respect to a continuous map $\gamma:[0,1] \rightarrow K \subseteq X$ satisfying $\gamma(0)=0$ and $\gamma^{\prime}\left(0^{+}\right) \in K$, then the necessary and sufficient conditions for optimality of $(P)$ are established.

\section{Introduction}

In recent years, there has been an increasing interest in studying the develpoment of optimality conditions for nondifferentiable multiobjective programming problems. Many authors established and employed some different Kuhn and Tucker type necessary conditions or other type necessary conditions to research optimal solutions; see [1-27] and references therein. In [7], Lai and Ho used the Pareto optimality condition to investigate multiobjective programming problems for semipreinvex functions. Lai [6] had obtained the necessary and sufficient conditions for optimality programming problems with semipreinvex assumptions. Some Pareto optimality conditions are established by Lai and Lin in [8]. Lai and Szilágyi [9] studied the programming with convex set functions and proved that the alternative theorem is valid for convex set functions defined on convex subfamily $S$ of measurable subsets in $X$ and showed that if the system

$$
\begin{gathered}
f(\Omega) \ll \theta, \\
g(\Omega)<\theta
\end{gathered}
$$

has on solution, where $\theta$ stands for zero vector in a topological vector space, then there exists a nonzero continuous linear function $\left(y^{*}, z^{*}\right) \in C^{*} \times D^{*}$ such that

$$
\left\langle f(\Omega), y^{*}\right\rangle+\left\langle g(\Omega), z^{*}\right\rangle \geq 0 \quad \forall \Omega \in S .
$$

In this paper, we study the following optimization problem:

$$
\begin{aligned}
& \min _{x \in K} \frac{f(x)}{g(x)} \\
& \text { subject to } \quad x \in K \subseteq X, \quad h_{i}(x) \leq 0, \\
& i=1,2, \ldots, m,
\end{aligned}
$$

where $K$ is a semiconnected subset in a locally convex topological vector space $X, f: K \rightarrow \mathbb{R}, g: K \rightarrow \mathbb{R}_{+}$and $h_{i}: K \rightarrow(-\infty, 0], i=1,2, \ldots, m$, are functions satisfying some suitable conditions. The purpose of this study is dealt with such constrained fractional semipreinvex programming problem. Finally, we established the Fritz John type necessary and sufficient conditions for the optimality of a fractional semipreinvex programming problem. 


\section{Preliminaries}

Throughout this paper, we let $X$ be a locally convex topological vector space over the real field $\mathbb{R}$. Denote $L^{1}(X)$ by the space of all linear operators from $X$ into $\mathbb{R}$.

Let $W$ be a nonempty convex subset of $X$. Let $f: W \rightarrow \mathbb{R}$ be differentiable at $x_{0} \in K$. Then there is a linear operator $A=f^{\prime}\left(x_{0}\right) \in L^{1}(X)$, such that

$$
\lim _{\alpha \rightarrow 0} \frac{f\left((1-\alpha) x_{0}+\alpha x\right)-f\left(x_{0}\right)}{\alpha}=f^{\prime}\left(x_{0}\right)\left(x-x_{0}\right) .
$$

Recall that a function $f: W \rightarrow \mathbb{R}$ is called convex on $W$, if

$$
f\left((1-\alpha) x_{0}+\alpha x\right) \leq(1-\alpha) f\left(x_{0}\right)+\alpha f(x)
$$

or

$$
\frac{f\left((1-\alpha) x_{0}+\alpha x\right)-f\left(x_{0}\right)}{\alpha} \leq f(x)-f\left(x_{0}\right) .
$$

If $f: W \rightarrow \mathbb{R}$ is convex and differentiable at $x_{0} \in K$, then by (3) and (5), we have

$$
f^{\prime}\left(x_{0}\right)\left(x-x_{0}\right) \leq f(x)-f\left(x_{0}\right) \text {. }
$$

In 1981, Hanson $[13,14]$ introduced a generalized convexity on $X$, so-called invexity; that is, $x-x_{0}$ is replaced by a vector $\tau\left(x_{0}, x\right) \in X$ in $(6)$, or

$$
f^{\prime}\left(x_{0}\right) \tau\left(x_{0}, x\right) \leq f(x)-f\left(x_{0}\right)
$$

So an invex function is indeed a generalization of a convex differentiable function.

Definition 1 (see [6]). (1) A set $K \subseteq X$ is said to be semiconnected with respect to a given $\tau: X \times X \rightarrow \mathbb{R}$ if

$$
x, y \in K, 0 \leq \alpha \leq 1 \Longrightarrow y+\alpha \tau(x, y, \alpha) \in K
$$

(2) A map $f: X \rightarrow \mathbb{R}$ is said to be semipreinvex on a semiconnected subset $K \subset X$ if each $(x, y, \alpha) \in K \times K \times[0,1]$ corresponds a vector $\tau(x, y, \alpha) \in X$ such that

$$
\begin{gathered}
f(x+\alpha \tau(x, y, \alpha)) \leq(1-\alpha) f(x)+\alpha f(y), \\
\lim _{\alpha \downarrow 0} \alpha \tau(x, y, \alpha)=\theta,
\end{gathered}
$$

where $\theta$ stands for the zero vector of $X$.

The following is an example of a bounded semiconnected set in $\mathbb{R}$, which is semiconnected with respect to a nontrivial $\tau$.
Example 2. Let $A:=[4,8], B:=[-8,-4]$ and $K:=A \cup B$ be bounded sets. Let $\tau: K \times K \times[0,1] \rightarrow \mathbb{R}$ be defined by

$$
\begin{aligned}
& \tau(x, y, \alpha)=\frac{x-y}{1-\alpha}, \quad \text { for }(x, y, \alpha) \in A \times A \times\left[0, \frac{1}{2}\right], \\
& \tau(x, y, \alpha)=\frac{x-y}{1-\alpha}, \quad \text { for }(x, y, \alpha) \in B \times B \times\left[0, \frac{1}{2}\right], \\
& \tau(x, y, \alpha)=\frac{-8-y}{1-\alpha}, \quad \text { for }(x, y, \alpha) \in A \times B \times\left[0, \frac{1}{2}\right], \\
& \tau(x, y, \alpha)=\frac{4-y}{1-\alpha}, \quad \text { for }(x, y, \alpha) \in B \times A \times\left[0, \frac{1}{2}\right], \\
& \tau(x, y, \alpha)=\frac{x-y}{\alpha}, \quad \text { for }(x, y, \alpha) \in A \times A \times\left[\frac{1}{2}, 1\right], \\
& \tau(x, y, \alpha)=\frac{x-y}{\alpha}, \quad \text { for }(x, y, \alpha) \in B \times B \times\left[\frac{1}{2}, 1\right], \\
& \tau(x, y, \alpha)=\frac{-8-y}{\alpha}, \quad \text { for }(x, y, \alpha) \in A \times B \times\left[\frac{1}{2}, 1\right], \\
& \tau(x, y, \alpha)=\frac{4-y}{\alpha}, \quad \text { for }(x, y, \alpha) \in B \times A \times\left[\frac{1}{2}, 1\right] .
\end{aligned}
$$

Then $K$ is a bound semiconnected set with respect to $\tau$.

Theorem 3 (see [6, Theorem 2.2]). Let $K \subset X$ be $a$ semiconnected subset and $f: K \rightarrow \mathbb{R}$ a semipreinvex map. Then any local minimum of $f$ is also a global minimum of $f$ over $K$.

From the assumption in problem 9, there exists a positive number $\lambda$ such that

$$
\begin{gathered}
\frac{f(y)}{g(y)} \geq \lambda \quad \forall y \in X, \\
f(y)-\lambda g(y) \geq 0 .
\end{gathered}
$$

Consequently, we can reduce the problem 9 to an equivalent nonfractional parametric problem:

$$
v(\lambda):=\min _{y \in X}(f(y)-\lambda g(y)) \geq 0
$$

where $\lambda \in[0, \infty)$ is a parameter.

We will prove that the problem $(P)$ is equivalent to the problem $\left(P_{\lambda^{*}}\right)$ for the optimal value $\lambda^{*}$. The following result is our main technique to derive the necessary and sufficient optimality conditions for problem $(P)$.

Theorem 4. Problem $(P)$ has an optimal solution $y_{0}$ with optimal value $\lambda^{*}$ if and only if $v\left(\lambda^{*}\right)=0$ and $y_{0}$ is an optimal solution of $\left(P_{\lambda^{*}}\right)$.

Proof. If $y_{0}$ is an optimal solution of $(P)$ with optimal value $\lambda^{*}$, that is,

$$
\lambda^{*}:=\frac{f\left(y_{0}\right)}{g\left(y_{0}\right)}=\min _{z \in X} \frac{f(z)}{g(z)} \leq \frac{f(z)}{g(z)} \quad \forall z \in X .
$$


It follows from (12) that

$$
\begin{gathered}
f(z)-\lambda^{*} g(z) \geq 0 \quad \forall z \in X, \\
f\left(y_{0}\right)-\lambda^{*} g\left(y_{0}\right)=0 .
\end{gathered}
$$

Thus, we have

$$
0 \leq \min _{z \in X}\left(f(z)-\lambda^{*} g(z)\right) \leq f\left(y_{0}\right)-\lambda^{*} g\left(y_{0}\right)=0 .
$$

Then, by (14), we get

$$
v\left(\lambda^{*}\right)=\min _{z \in X}\left(f(z)-\lambda^{*} g(z)\right)=f\left(y_{0}\right)-\lambda^{*} g\left(y_{0}\right)=0 .
$$

Therefore, $y_{0}$ is an optimal solution of $\left(P_{\lambda^{*}}\right)$ and $v\left(\lambda^{*}\right)=0$.

Conversely, if $y_{0}$ is an optimal solution of $\left(P_{\lambda^{*}}\right)$ with optimal value $v\left(\lambda^{*}\right)=0$, then

$$
f\left(y_{0}\right)-\lambda^{*} g\left(y_{0}\right)=\min _{z \in X}\left(f(z)-\lambda^{*} g(z)\right)=0 .
$$

So

$$
f(z)-\lambda^{*} g(z) \geq 0=f\left(y_{0}\right)-\lambda^{*} g\left(y_{0}\right) \quad \forall z \in X .
$$

It follows from (17) that

$$
\begin{gathered}
\frac{f(z)}{g(z)} \geq \lambda^{*} \quad \forall z \in X, \\
\frac{f\left(y_{0}\right)}{g\left(y_{0}\right)}=\lambda^{*},
\end{gathered}
$$

and hence

$$
\begin{gathered}
\min _{z \in X} \frac{f(z)}{g(z)} \geq \lambda^{*}, \\
\min _{z \in X} \frac{f(z)}{g(z)} \leq \frac{f\left(y_{0}\right)}{g\left(y_{0}\right)}=\lambda^{*} .
\end{gathered}
$$

Therefore,

$$
\min _{z \in X} \frac{f(z)}{g(z)}=\lambda^{*}=\frac{f\left(y_{0}\right)}{g\left(y_{0}\right)}
$$

and we know $y_{0}$ is an optimal solution of $(P)$ with optimal value $\lambda^{*}$.

\section{The Existence of the Necessary and Sufficient Conditions for Semipreinvex Functions}

Definition 5 (see [6]). A mapping $f: K \subset X \rightarrow \mathbb{R}$ is said to be arcwise directionally (in short, arc-directionally) differentiable at $x_{0} \in K$ with respect to a continuous arc $\beta:[0,1] \rightarrow K \subset X$ if $x_{0}+\beta(t) \in K$ for $t \in[0,1]$ with

$$
\beta(0)=\theta, \quad \beta^{\prime}\left(0^{+}\right)=u \quad(\text { in } X),
$$

that is, the continuous function $\beta$ is differentiable from right at 0 , and the limit

$$
\lim _{t \downarrow 0} \frac{f\left(x_{0}+\beta(t)\right)-f\left(x_{0}\right)}{t} \cong f^{\prime}\left(x_{0} ; u\right) \text { exists. }
$$

Note that the arc directional derivative $f^{\prime}\left(x_{0} ;\right)$ is a mapping from $X$ into $\mathbb{R}$. Moreover, how can we make $K$ to be a semiconnected set? Indeed, we can construct a function $\tau$ concerned with $\beta$ defined as follows.

For any $x, y \in K$ and $t \in[0,1]$, we choose a vector

$$
\tau(x, y, t):=\frac{\beta(t)}{t}=\frac{\beta(t)-\beta(0)}{t-0},
$$

then

$$
\begin{gathered}
\lim _{t \downarrow 0} \tau(x, y, t)=\beta^{\prime}\left(0^{+}\right)=u, \\
\left.\frac{d}{d t}[t \tau(x, y, t)]\right|_{t=0^{+}}=\beta^{\prime}\left(0^{+}\right)=u .
\end{gathered}
$$

Let $f: X \rightarrow \mathbb{R},-g: X \rightarrow \mathbb{R}_{-}$and $h_{i}: X \rightarrow \mathbb{R}_{-}, i=$ $1,2, \ldots, m$, be semipreinvex maps on a semiconnected subset $K$ in $X$. Consider a constrained programming problem as $(P)$.

The following Fritz John type theorem is essential in this section for programming problem $(P)$.

Theorem 6 (Necessary Optimality Condition). Suppose that $f,-g$ and $h_{i}, i=1,2, \ldots, m$ are arc-directionally differentiable at $x_{0} \in K$ and semipreinvex on $K$ with respect to a continuous arc $\beta$ defined as in Definition 5. If $x_{0}$ minimizes locally for the semipreinvex programming problem $(P)$, then there exist $\lambda^{*} \in$ $(0, \infty)$ and $\left\{\gamma_{i}\right\}_{i=1}^{m} \subseteq[0, \infty)$ such that

$$
f^{\prime}\left(x_{0} ; u\right)-\lambda^{*} g^{\prime}\left(x_{0} ; u\right)+\sum_{i=1}^{m} \gamma_{i} h_{i}^{\prime}\left(x_{0} ; u\right) \geq 0
$$

where $u=\beta^{\prime}\left(0^{+}\right)$and

$$
\sum_{i=1}^{m} \gamma_{i} h_{i}\left(x_{0}\right)=0
$$

Proof. By Theorem 4, the minimum solution to $(P)$ is also a minimum to $\left(P_{\lambda^{*}}\right)$. Then $x_{0}$ is the local minimal solution to $\left(P_{\lambda^{*}}\right)$. By Theorem 3, we have $x_{0}$ is the global minimal solution to $\left(P_{\lambda}\right)$. It follows that the system

$$
\begin{gathered}
{\left[f(x)-\lambda^{*} g(x)\right]-\left[f\left(x_{0}\right)-\lambda^{*} g\left(x_{0}\right)\right]<0,} \\
h_{i}(x) \leq 0, \quad i=1,2, \ldots, m
\end{gathered}
$$

has no solution in $K$, then we have

$$
\left[f(x)-\lambda^{*} g(x)\right]-\left[f\left(x_{0}\right)-\lambda^{*} g\left(x_{0}\right)\right]+\sum_{i=1}^{m} \gamma_{i} h_{i}(x)<0
$$

has no solution in $K$ for any $\left\{\gamma_{i}\right\}_{i=1}^{m} \subseteq[0, \infty)$. Thus for any $x \in K$,

$$
\left[f(x)-\lambda^{*} g(x)\right]-\left[f\left(x_{0}\right)-\lambda^{*} g\left(x_{0}\right)\right]+\sum_{i=1}^{m} \gamma_{i} h_{i}(x) \geq 0
$$


for some $\left\{\gamma_{i}\right\}_{i=1}^{m} \subseteq[0, \infty)$. Putting $x=x_{0}$ in (29), we get

$$
\sum_{i=1}^{m} \gamma_{i} h_{i}\left(x_{0}\right) \geq 0
$$

Since $\gamma_{i} \geq 0$ and $h_{i}\left(x_{0}\right) \leq 0$, it follows that

$$
\sum_{i=1}^{m} \gamma_{i} h_{i}\left(x_{0}\right)=0
$$

So (26) is proved.

As $K$ is a semiconnected set, for any $x \in K$ and $t \in[0,1]$, we have

$$
x_{0}+t \tau\left(x_{0}, x, t\right) \in K
$$

For $t \neq 0$, the point $\tilde{x}=x_{0}+t \tau\left(x_{0}, x, t\right) \neq x_{0}$ does not solve the system (27). So substituting $\tilde{x}$ in (29) and using the result (26), we obtain

$$
\begin{aligned}
& {\left[f\left(x_{0}+t \tau\left(x_{0}, x, t\right)\right)-f\left(x_{0}\right)\right]} \\
& \quad-\lambda^{*}\left[g\left(x_{0}+t \tau\left(x_{0}, x, t\right)\right)-g\left(x_{0}\right)\right] \\
& \quad+\sum_{i=1}^{m} \gamma_{i}\left(h_{i}\left(x_{0}+t \tau\left(x_{0}, x, t\right)\right)-h_{i}\left(x_{0}\right)\right) \geq 0 .
\end{aligned}
$$

Since $f$ and $g$ are arc-directionally differentiable with respect to $\beta$, choose a vector $\tau\left(x_{0}, x, t\right)$ as $(23)$, so that (24) hold. It follows that if we divide (33) by $t \neq 0$ and take the limit as $t \downarrow 0$, then we have

$$
f^{\prime}\left(x_{0} ; u\right)-\lambda^{*} g^{\prime}\left(x_{0} ; u\right)+\sum_{i=1}^{m} \gamma_{i} h_{i}^{\prime}\left(x_{0} ; u\right) \geq 0,
$$

which proves (25) and the proof of theorem is completed.

Theorem 7 (Sufficient Optimality Condition). Let $f,-g$ and $h_{i}, i=1,2, \ldots, m$ be arc-directionally differentiable at $x_{0} \in$ $K$ and semipreinvex on $K$ with respect to a continuous arc $\beta$ defined as in Definition 5. If there exist $\lambda \in(0, \infty)$ and $\left\{\gamma_{i}\right\}_{i=1}^{m} \subseteq$ $[0, \infty)$ satisfying

$$
f^{\prime}\left(x_{0} ; u\right)-\lambda g^{\prime}\left(x_{0} ; u\right)+\sum_{i=1}^{m} \gamma_{i} h_{i}^{\prime}\left(x_{0} ; u\right) \geq 0,
$$

with $u=\beta^{\prime}\left(0^{+}\right)$and

$$
\sum_{i=1}^{m} \gamma_{i} h_{i}\left(x_{0}\right)=0
$$

then $x_{0}$ is an optimal solution for problem $(P)$.

Proof. Suppose to the contrary that $x_{0}$ is not optimal for problem $(P)$ and $\lambda=f\left(x_{0}\right) / g\left(x_{0}\right)$. Then $f\left(x_{0}\right)-\lambda g\left(x_{0}\right)=0$. Therefore,

$$
0 \leq \min _{x \in X}(f(x)-\lambda g(x)) \leq f\left(x_{0}\right)-\lambda g\left(x_{0}\right)=0,
$$

thus $v(\lambda)=\min _{x \in X}(f(x)-\lambda g(x))=0$.
By Theorem $4, x_{0}$ was not optimal for problem $\left(P_{\lambda}\right)$. Then there is an $x \in X$ such that

$$
\begin{gathered}
f(x)-\lambda g(x)<f\left(x_{0}\right)-\lambda g\left(x_{0}\right), \\
h_{i}(x) \leq 0
\end{gathered}
$$

for $i=1,2, \ldots, m$. Moreover, we have

$$
\begin{gathered}
{[f(x)-\lambda g(x)]-\left[f\left(x_{0}\right)-\lambda g\left(x_{0}\right)\right]<0} \\
\sum_{i=1}^{m} \gamma_{i}\left[h_{i}(x)-h_{i}\left(x_{0}\right)\right] \leq 0 \quad\left(\text { since } \sum_{i=1}^{m} \gamma_{i} h_{i}\left(x_{0}\right)=0\right)
\end{gathered}
$$

for any $\left\{\gamma_{i}\right\}_{i=1}^{m} \subseteq[0, \infty)$. Thus

$$
\begin{gathered}
{[f(x)-\lambda g(x)]-\left[f\left(x_{0}\right)-\lambda g\left(x_{0}\right)\right]} \\
+\sum_{i=1}^{m} \gamma_{i}\left[h_{i}(x)-h_{i}\left(x_{0}\right)\right]<0 .
\end{gathered}
$$

Since the semi-preinvex maps $f,-g$ and $h_{i}, i=1,2, \ldots, m$ are arc-directionally differentiable, it follows that for $\left(x, x_{0}, t\right) \in K \times K \times[0,1]$ there corresponds a vector $\tau\left(x, x_{0}, t\right) \in X$ such that

$$
\begin{gathered}
f\left(x_{0}+t \tau\left(x, x_{0}, t\right)\right) \leq(1-t) f\left(x_{0}\right)+t f(x), \\
-g\left(x_{0}+t \tau\left(x, x_{0}, t\right)\right) \leq(1-t)(-g)\left(x_{0}\right)+t(-g)(x), \\
h_{i}\left(x_{0}+t \tau\left(x, x_{0}, t\right)\right) \leq(1-t) h_{i}\left(x_{0}\right)+t h_{i}(x),
\end{gathered}
$$

and so

$$
\begin{gathered}
\frac{f\left(x_{0}+t \tau\left(x, x_{0}, t\right)\right)-f\left(x_{0}\right)}{t} \leq f(x)-f\left(x_{0}\right), \\
\frac{(-g)\left(x_{0}+t \tau\left(x, x_{0}, t\right)\right)+g\left(x_{0}\right)}{t} \leq(-g)(x)+g\left(x_{0}\right), \\
\frac{h_{i}\left(x_{0}+t \tau\left(x, x_{0}, t\right)\right)-h_{i}\left(x_{0}\right)}{t} \leq h_{i}(x)-h_{i}\left(x_{0}\right) .
\end{gathered}
$$

Letting $t \downarrow 0$, we have $\lim _{t \downarrow 0} \tau\left(x, x_{0}, t\right)=\beta^{\prime}\left(0^{+}\right)=u$ and the last inequalities imply

$$
\begin{gathered}
f^{\prime}\left(x_{0}, u\right) \leq f(x)-f\left(x_{0}\right), \\
-g^{\prime}\left(x_{0}, u\right) \leq-\left[g(x)-g\left(x_{0}\right)\right], \\
h_{i}^{\prime}\left(x_{0}, u\right) \leq h_{i}(x)-h_{i}\left(x_{0}\right) .
\end{gathered}
$$

Consequently, from (41) and (44), we obtain

$$
f^{\prime}\left(x_{0} ; u\right)-\lambda g^{\prime}\left(x_{0} ; u\right)+\sum_{i=1}^{m} \gamma_{i} h_{i}^{\prime}\left(x_{0} ; u\right)<0,
$$

which contradicts the fact of (35). Therefore $x_{0}$ is an optimal solution of problem $(P)$. 
Since any global minimal is a local minimal, applying Theorems 6 and 7, we can obtain the necessary and sufficient conditions for problem $(P)$.

Theorem 8. Suppose that $f,-g$ and $h_{i}, i=1,2, \ldots, m$ are arcdirectionally differentiable at at $x_{0} \in K$ and semi-preinvex on $K$ with respect to a continuous arc $\beta$ defined as in Definition 5. If $x_{0}$ minimizes globally for the semi-preinvex programming problem $(P)$ if and only if there exists $\left(\lambda, \gamma_{i}\right) \in \mathbb{R}^{+} \times\left(\mathbb{R}^{+} \cup\{0\}\right)$, $i=1,2, \ldots, m$, such that

$$
f^{\prime}\left(x_{0} ; u\right)-\lambda g^{\prime}\left(x_{0} ; u\right)+\sum_{i=1}^{m} \gamma_{i} h_{i}^{\prime}\left(x_{0} ; u\right) \geq 0,
$$

where $u=\beta^{\prime}\left(0^{+}\right)$and

$$
\sum_{i=1}^{m} \gamma_{i} h_{i}\left(x_{0}\right)=0
$$

Remark 9. Our results also hold for preinvex functions.

\section{Acknowledgments}

The research of Wei-Shih Du was supported partially under Grant no. NSC 101-2115-M-017-001 by the National Science Council of the Republic of China.

\section{References}

[1] B. D. Craven, "Invex functions and constrained local minima," Bulletin of the Australian Mathematical Society, vol. 24, no. 3, pp. 357-366, 1981.

[2] B. D. Craven and B. M. Glover, "Invex functions and duality," Australian Mathematical Society, vol. 39, no. 1, pp. 1-20, 1985.

[3] F. H. Clarke, Optimization and Nonsmooth Analysis, Canadian Mathematical Society Series of Monographs and Advanced Texts, John Wiley \& Sons, New York, NY, USA, 1983.

[4] F. H. Clarke, Optimization and Nonsmooth Analysis, John Wiley \& Sons, New York, NY, USA, 2nd edition, 1983.

[5] H. Dietrich, "On the convexification procedure for nonconvex and nonsmooth infinite-dimensional optimization problems," Journal of Mathematical Analysis and Applications, vol. 161, no. 1, pp. 28-34, 1991.

[6] H.-C. Lai, "Optimality conditions for semi-preinvex programming," Taiwanese Journal of Mathematics, vol. 1, no. 4, pp. 389404, 1997.

[7] H. C. Lai and C. P. Ho, "Duality theorem of nondifferentiable convex multiobjective programming," Journal of Optimization Theory and Applications, vol. 50, no. 3, pp. 407-420, 1986.

[8] H.-C. Lai and L.-J. Lin, "Moreau-Rockafellar type theorem for convex set functions," Journal of Mathematical Analysis and Applications, vol. 132, no. 2, pp. 558-571, 1988.

[9] H. C. Lai and P. Szilágyi, "Alternative theorems and saddlepoint results for convex programming problems of set functions with values in ordered vector spaces," Acta Mathematica Hungarica, vol. 63, no. 3, pp. 231-241, 1994.

[10] H. C. Lai and J. C. Liu, "Minimax fractional programming involving generalised invex functions," The ANZIAM Journal, vol. 44, no. 3, pp. 339-354, 2003.
[11] J.-C. Chen and H.-C. Lai, "Fractional programming for variational problem with $F, \rho, \theta$-invexity," Journal of Nonlinear and Convex Analysis, vol. 4, no. 1, pp. 25-41, 2003.

[12] J. C. Liu, "Optimality and duality for generalized fractional programming involving nonsmooth $(F, \rho)$-convex functions," Computers and Mathematics with Applications, vol. 42, pp. 437446, 1990.

[13] M. A. Hanson, "On sufficiency of the Kuhn-Tucker conditions," Journal of Mathematical Analysis and Applications, vol. 80, no. 2, pp. 545-550, 1981.

[14] M. A. Hanson and B. Mond, "Necessary and sufficient conditions in constrained optimization," Mathematical Programming, vol. 37, no. 1, pp. 51-58, 1987.

[15] S. K. Mishra and G. Giorgi, Invexity and Optimization, vol. 88 of Nonconvex Optimization and Its Applications, Springer, Berlin, Germnay, 2008.

[16] S. K. Mishra, S. Wang, and K. K. Lai, V-Invex Functions and Vector Optimization, vol. 14 of Springer Optimization and Its Applications, Springer, New York, NY, USA, 2008.

[17] S. K. Mishra, S.-Y. Wang, and K. K. Lai, Generalized Convexity and Vector Optimization, vol. 90 of Nonconvex Optimization and Its Applications, Springer, Berlin, Germnay, 2009.

[18] S. K. Mishra and V. Laha, "On approximately star-shaped functions and approximate vector variational inequalities," Journal of Optimization Theory and Applications. In press.

[19] S. K. Mishra and V. Laha, "On V-r-invexity and vector variational Inequalities," Filomat, vol. 26, no. 5, pp. 1065-1073, 2012.

[20] S. K. Mishra and B. B. Upadhyay, "Nonsmooth minimax fractional programming involving $\eta$-pseudolinear functions," Optimization. In press.

[21] S. K. Mishra and M. Jaiswal, "Optimality conditions and duality for nondifferentiable multiobjective semi-infinite programming," Vietnam Journal of Mathematics, vol. 40, pp. 331343, 2012.

[22] N. G. Rueda and M. A. Hanson, "Optimality criteria in mathematical programming involving generalized invexity," Journal of Mathematical Analysis and Applications, vol. 130, no. 2, pp. 375-385, 1988.

[23] T. W. Reiland, "Nonsmooth invexity," Bulletin of the Australian Mathematical Society, vol. 12, pp. 437-446, 1990.

[24] T. Weir, "Programming with semilocally convex functions," Journal of Mathematical Analysis and Applications, vol. 168, no. 1, pp. 1-12, 1992.

[25] T. Weir and V. Jeyakumar, "A class of nonconvex functions and mathematical programming," Bulletin of the Australian Mathematical Society, vol. 38, no. 2, pp. 177-189, 1988.

[26] T. Antczak, "( $p, r)$-invex sets and functions," Journal of Mathematical Analysis and Applications, vol. 263, no. 2, pp. 355-379, 2001.

[27] Y. L. Ye, "D-invexity and optimality conditions," Journal of Mathematical Analysis and Applications, vol. 162, no. 1, pp. 242249, 1991. 


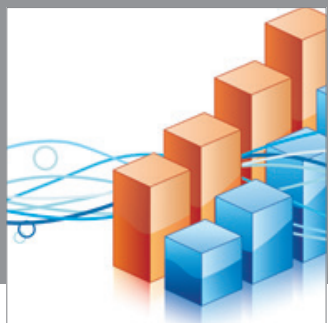

Advances in

Operations Research

mansans

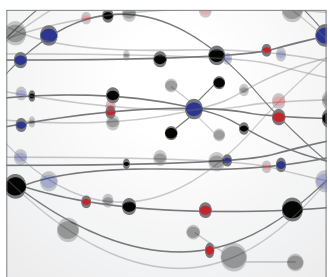

The Scientific World Journal
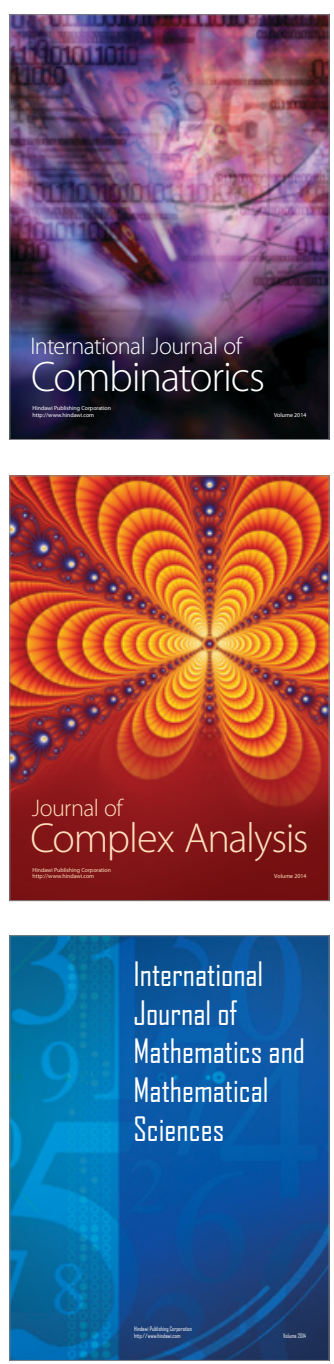
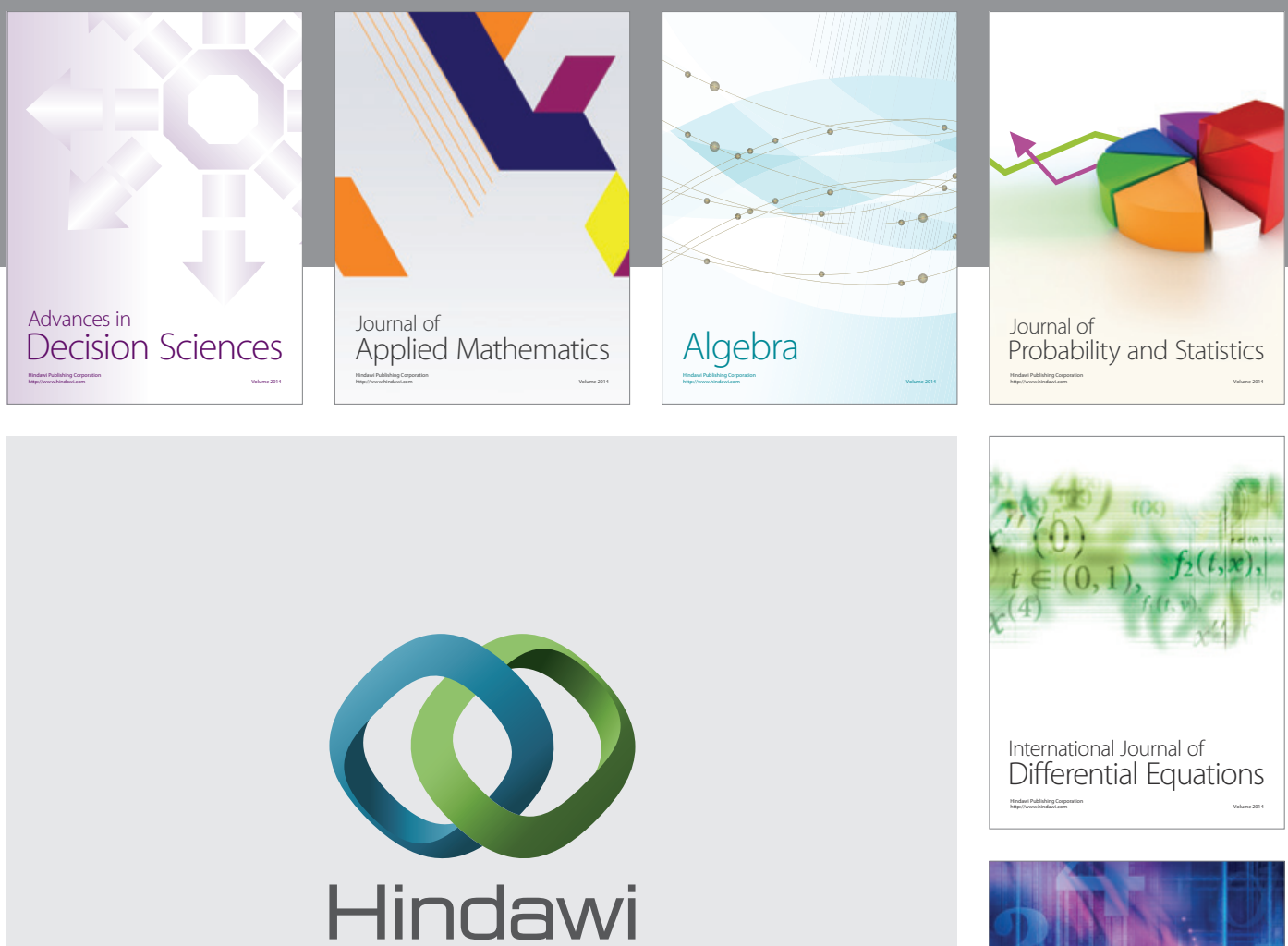

Submit your manuscripts at http://www.hindawi.com
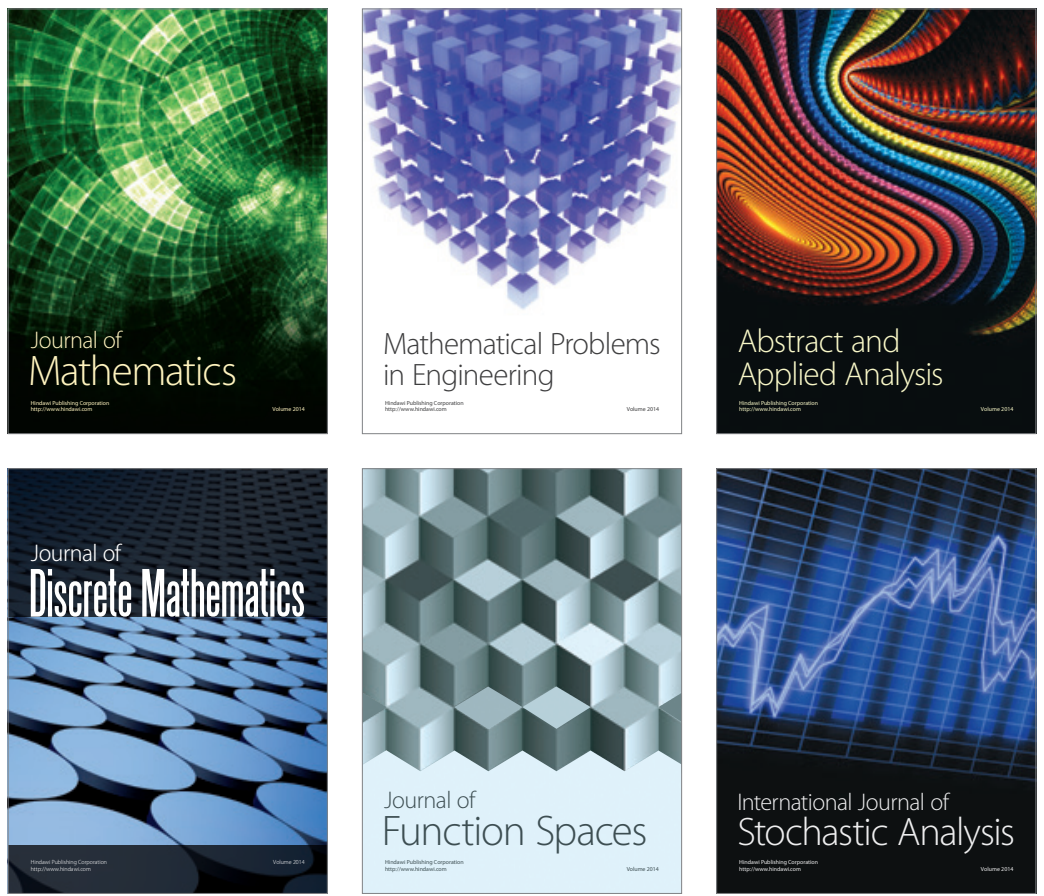

Journal of

Function Spaces

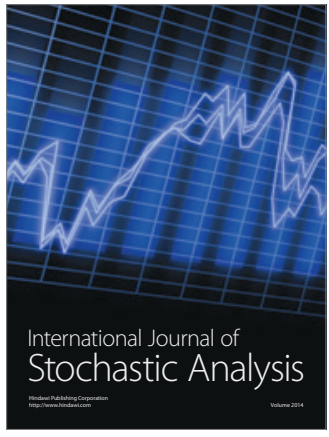

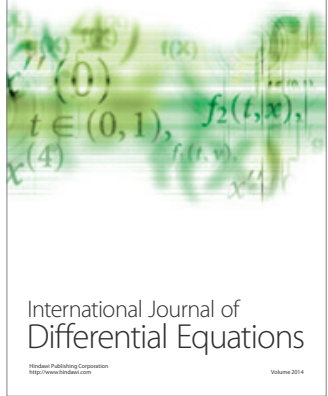
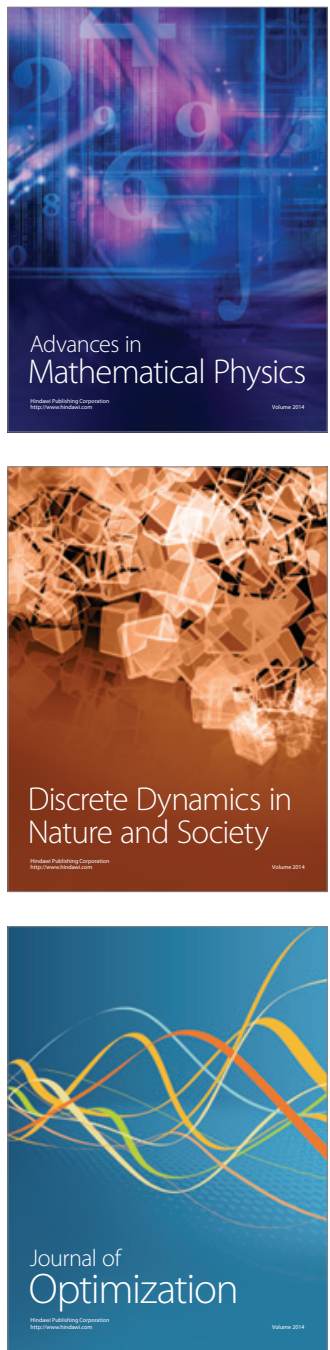